\title{
THE SIMULATION METHOD FOR THE IDENTIFICATION THE SURFACE IRREGULARITIES
}

\author{
D. Gogolewski*
}

\begin{abstract}
The main purpose of the research was to assess the possibility of application of the twodimensional wavelet analysis to perform simulations to detect the characteristic surface areas. The research was performed on the basis of the possibility of assessment of the parameters of the face milling process, i.e. feed per revolution and feed per tooth. The images of the surface layer of the samples were analyzed. The analysis of the images obtained after the wavelet filtration showed that in the images were visible characteristic bands, which were created as a result of removing material by particular cutting inserts. The application of the wavelet analysis allowed to detect characteristic information about surface attributes, which were not visible before the filtration process.
\end{abstract}

Keywords: wavelet transform, feed per tooth, face milling, geometrical product specification.

\section{Introduction}

The dynamic development of science and technology implies an increasing requirements for the quality of machine parts. There are many methods of production of machine parts. However, milling process is one of the most commonly used in the production process. The application of numerical methods and computer techniques cause that to diagnosis of the milling process are used more and more complex algorithms. These methods allow to characterize 3D signals and detect in them relevant information. The classical methods of analysis are increasingly replaced by new data processing algorithms, which were developed at the turn of recent years. When applying these techniques to signal analysis, it is possible to better understand real occurrence of certain phenomena occurring, among others, in the machining process. Those phenomena should be treated as non-stationary signals, therefore appropriate algorithms should be used to evaluate them.

The analysis of the surface texture of machine parts is crucial part in the manufacturing process. For this purpose, sophisticated algorithms are used to highlight important information about the signals. The application of two-dimensional wavelet transform allows to complex analysis of the surface texture (Zahouani et al., 2008). The wavelet analysis was developed at the turn of the twentieth and twenty-first century. This method allows to more complex assessment of the measurement data. Analyzing signal using a wavelet transform it is possible to detect and to provide information about irregularities or sudden changes in the signal, as well as to determine the place of their occurrence. This fact makes that it is possible to use this method of filtration for the diagnostic analysis of the 3D surface topography. Wavelet transform is mainly used to analysis of non-stationary signals, that is, those which are characterized by non-periodic irregularities. The application of wavelet transform leads to removing signal background features. On the subsequent level of decomposition are analyzed information on different frequencies. Thus, it is possible, on particular level of analysis to detect characteristic information, which is not visible at other decomposition level. Wavelet transform uses a wide range of mother wavelets. Each group has different properties of wavelets and the appropriate wavelet choice is important for further signal analysis. Various mother wavelets can be successfully used for surface analysis. However, the results obtained for a particular mother wavelets at subsequent levels of decomposition are significantly different from each

PhD. Damian Gogolewski: Chair of Mechanical Technology and Metrology, Kielce University of Technology, Al. 1000-lecia P. P. 7, 25-314 Kielce; PL dgogolewski@tu.kielce.pl 
other. The selection of the mother wavelet can be made based on the statistical tests presented in the paper (Stępien, et al., 2015). Many researchers have used wavelet transform to diagnose milling process (Zhai, et al., 2011, Romero-Troncoso, et al., 2004, Kasashima, 1994) or to analyze the Geometrical Product Specifications signals. In (Abdul-Rahman, 2013) authors applied wavelet transform to filtering of freeform surfaces. In the work (Stępień, 2013) were analyzed the deviations of cylindrical surfaces. The work (Wang, et al., 2010) present results of the application of wavelet transform to assess nanotopography of crystal surfaces. In the work (Jiang, 2008) a engineering and biomedical surfaces were used to demonstrate the application of wavelet analysis in the assessment of surface topographical features. Zawada-Tomkiewicz (2010) applied wavelet transform to describe a digital image of a machined surface. The theory and techniques for the extraction of morphological structures from micro/nano scalar surfaces were investigated in (Jiang, 2004). Analyzing the current state of art it can be noted that wavelet transform is appropriate tool to make the filtration process of the surface texture, however is still rarely used tool.

The issue of shaping of the surface layer in machining processes is undoubtedly a complex process, which is related with influence of particular machining errors. These errors are caused by periodically variable rigidity of system which consist with machine tool - handle - workpiece - tool, condition of cutting inserts or pockets in the main body of the milling cutter, improperly selected process parameters or insertion errors of cutting inserts (Nowakowski, 2017). Both axial as well as radial errors in the positioning of the cutting inserts affect the actual values of the milling process parameters. Radial errors have a significant influence on the value of the feed per tooth parameter, while axial errors affect on the depth of cut (Nowakowski, 2014). Therefore, it should be stated that the feed per tooth parameter is one of the most important factor which influence on the surface roughness.

\section{Methods}

The main aim of the research was to assess the possibility of application wavelet analysis to evaluate the value of selected parameters of the face milling process, i.e. feed per revolution and feed per tooth. For this purpose there were produced sixty-four samples. The input parameters of the face milling process were: cutting depth in the range of $a_{p}=0.1-2 \mathrm{~mm}$, feed per tooth in the range of $f_{z}=0.1-0.22 \mathrm{~mm} /$ tooth and cutting speed in the range of $v_{c}=200-350 \mathrm{~m} / \mathrm{min}$. The vertical milling centre AVIA VMC 800 was employed to carry out cutting process. Measurements were performed using the optical profilometer Talysurf CCI. The research was carried out on the images of the surface roughness. All signals were analyzed using selected mother wavelets. Due to the multiplicity of data, the results of analysis for three selected samples were presented in the article. The machining parameters are presented in Table 1.

Tab. 1: Milling process parameters.

\begin{tabular}{cccc}
\hline No. sample & $\boldsymbol{v}_{\boldsymbol{c}}, \mathbf{m} / \mathbf{m i n}$ & $\boldsymbol{f}_{\boldsymbol{z}}, \mathbf{m m} / \mathbf{t o o t h}$ & $\boldsymbol{a}_{\boldsymbol{p}}, \mathbf{m m}$ \\
\hline 17 & 250 & 0.1 & 0.1 \\
\hline 44 & 300 & 0.18 & 2 \\
\hline 59 & 350 & 0.18 & 1 \\
\hline
\end{tabular}

The results of the analysis for particular samples are presented in Figures 1-3. In Figures $1 b, 2 b$ and $3 b$, the distances which correspond the set parameter value of feed per tooth have been marked. These distances correspond to the detected distances of the particular, characteristic bands, which were identified in presented Figures.

In Figure 1a is presented the view of the surface roughness for sample No. 44. The analysis of the image showed that there were not possible in unambiguous way to detect in image the characteristic surface zones, which were created as a result of removing material by particular cutting inserts. On this image it can be noted two characteristic bands which represent zones of impact one and the same cutting insert. It should be noted that this cutting insert was positioned incorrectly. The filtered surface image which was obtained using mother wavelet db20 is presented in Figure $1 \mathrm{~b}$. In the signal generated as a result of the wavelet-transform-based decomposition of the surface roughness signal it is possible to detect machining marks for all five cutting inserts. The remove a part of surface irregularities allow to visualize characteristic information which were not visible before filtration process. The distance between each 
cutting marks correspond to the specified parameter value of the feed per tooth. Therefore, it can be concluded that the application the wavelet analysis to signal filtration allows to achieve satisfactory, better results.
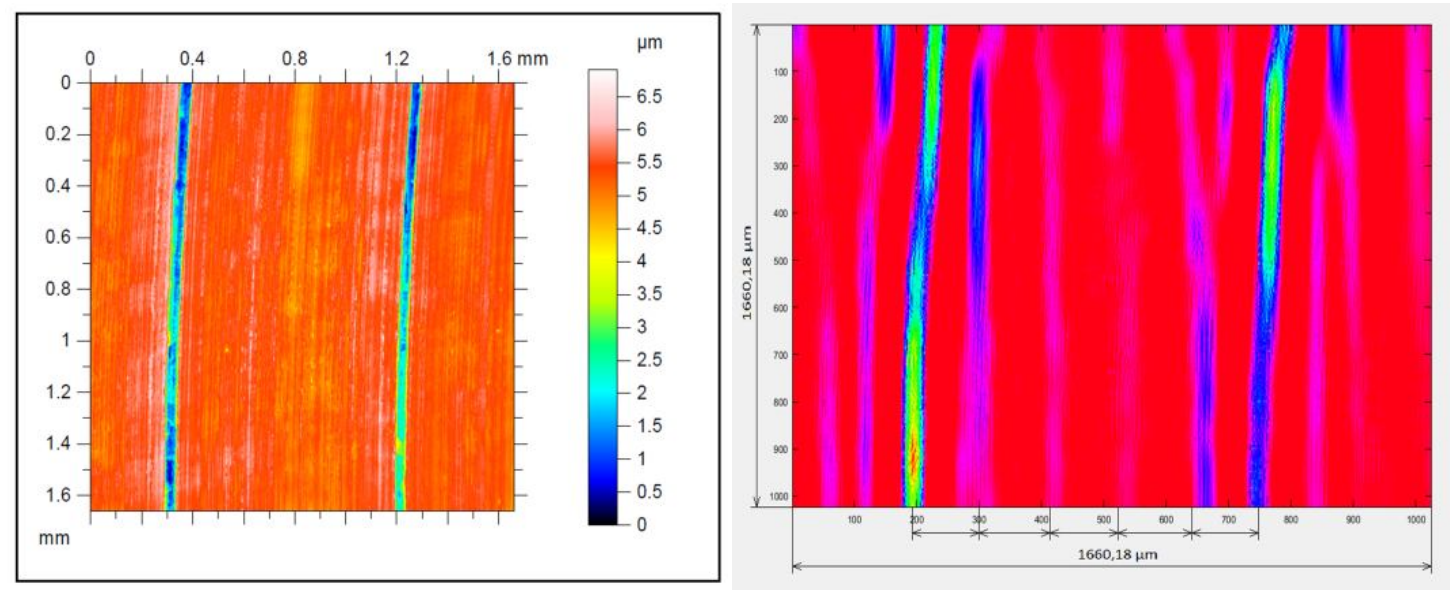

Fig. 1: Sample No. 44 a) surface roughness b) filtered surface image

An analogous analysis was also carried out for other samples. In Figure 2 is presented the results of analysis for sample No. 17. The surface is characterized by a regular distribution of irregularities. The analysis performed using mother wavelet $\mathrm{db} 15$ showed that in the filtered image were created characterisitc bands which represent particular cutting marks.
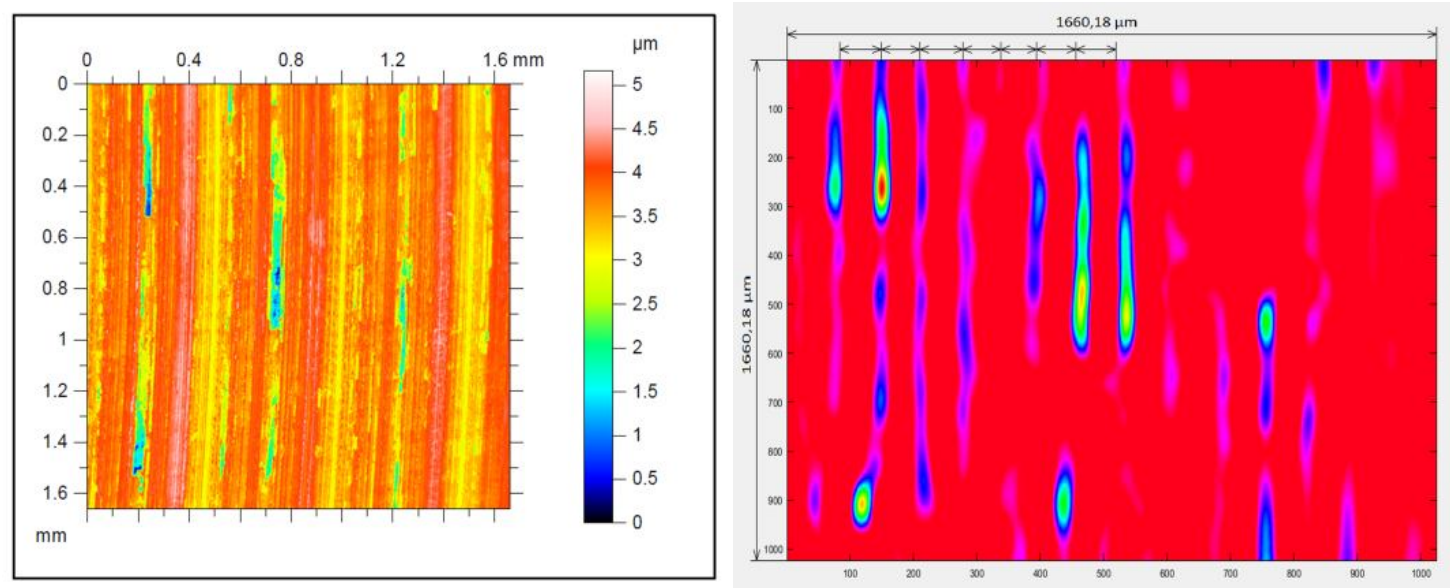

Fig. 2: Sample No. 17 a) surface roughness b) filtered surface image

The analysis was also carried out for surfaces of the sample where machining marks were observed in various directions. This machining marks indicate that the material was removed by the rearward flank of cutting insert. Figure 3 shows the results of filtration for sample No. 59 and mother wavelet coif5. The application of wavelet analysis resulted in the removal of a part of surface irregularities. From the surface was filtered out components in a certain frequency range. In this figure, particular machining marks were highlight. 

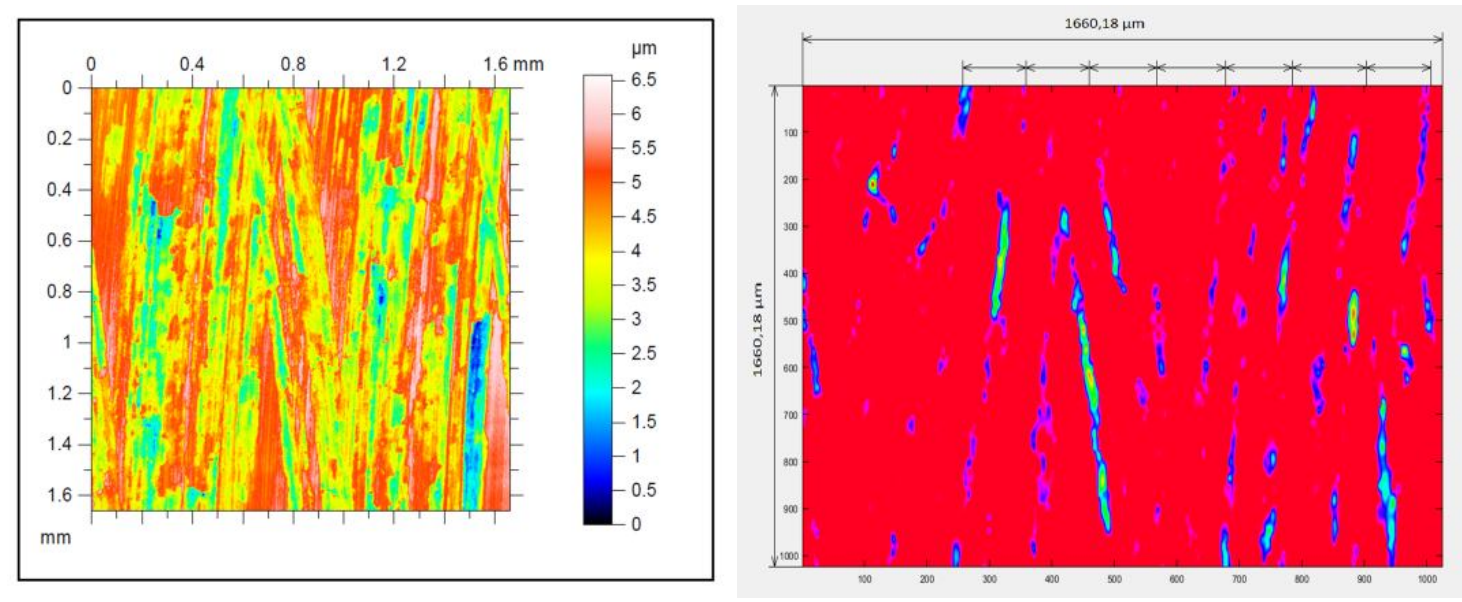

Fig. 3: Sample No. 59 a) surface roughness b) filtered surface image

\section{Conclusions}

The research result shows that it is possible to apply two-dimensional wavelet transform to analysis the surface topography signal. The research was carried out in order to identify value of the feed per revolution and feed per tooth parameter. Based on the obtained results, it can be concluded that wavelet analysis is an appropriate tool for assessing value of selected parameters of the face milling process. In the created signals, it can be detected characteristic information which are not visible on the surface topography images, before filtration process. On the tested surfaces were identified bands, which represent the locus of material removing by particular cutting inserts. The research was performed for various surface topography signals. There were analyzed both image of samples on which the surface irregularities were periodical, as well as for the samples image on which the cutting process did not cause visible, regular impact of each cutting insert on the surface layer. In all cases, on the obtained images it was possible to detect crucial information about signal.

\section{References}

Abdul-Rahman H S, Jiang X and Scott P (2013) Freeform surface filtering using the lifting wavelet transform Precision Engineering, 37 pp. 187-202

Jiang X., Blunt L. (2004) Third generation wavelet for the extraction of morphological features from micro and nano scalar surface, Wear, 257, 1235-1240

Jiang, X., Scott P. and Whitehouse D. (2008) Wavelet and their applications for surface metrology, CIRP Annals Manufacturing Technology, 57, pp. 555-558.

Kasashima N., Mori K. and, Herrera-Ruiz G. (1994) Diagnosing cutting tool condition in milling using wavelet transform, Advancement of Intelligent Production, pp. 339-344.

Nowakowski, Ł. (2014) The influence of selected parameters of face milling process on the geometric structure surfaces $\mathrm{PhD}$ dissertation, Kielce.

Nowakowski, Ł., Miko E. and Skrzyniarz M (2017) Milling with a tool with unevenly distributed cutting plates. In: Engineering Mechanics 2017, Brno University of Technology, Brno, pp. 726-729.

Romero-Troncoso R.J., Herrera-Ruiz G., Terol-Villalobos I. and Jauregui-Correa J.C. (2004) FPGA based on-line tool breakage detection system for CNC milling machines, Mechatronics, 14, pp. 439-454.

Stępien K. and Makieła W. (2013) An analysis of deviations of cylindrical surfaces with the use of wavelet transform, Metrology and Measurement System 20, 1, pp. 139-150

Stępien K., Makieła W., Stoic A. and Samardzic I. (2015) Defining the criteria to select the wavelet type for the assessment of surface quality. Tehnicki Vjesnik- Technical Gazette, 22, 3, pp. 781-784.

Wang H. X., Zong W. J., Sun T. and Liu Q. (2010) Modification of three dimensional topography of the machined KDP crystal surface using wavelet analysis method, Applied Surface Science, 256, 16,pp. 5061-5068

Zahouani H., Mezghani S., Vargiolu R. and Dursapt M. (2008) Identification of manufacturing signature by 2D wavelet decomposition. Wear, 264, pp. 480-485.

Zawada-Tomkiewicz A. (2010) Estimation of surface roughness parameter based on machined surface image. Metrology and Measurement System, 17, 3, pp. 493-504.

Zhai L.Y., Er M.J., Li X., Gan O.P. and San L. (2011) Wavelet-based analysis of force signal for monitoring and prediction of cutting performance in high-speed milling processes Engineering Letters, 19, pp. 155-158. 